\title{
Analysis of the factors affecting the safety of robotic stereotactic body radiation therapy for hepatocellular carcinoma patients
}

\author{
Xiaojie Liu',* \\ Yongchun Songl,* \\ Ping Liang ${ }^{2}$ \\ Tingshi Su ${ }^{2}$ \\ Huojun Zhang ${ }^{3}$ \\ Xianzhi Zhao ${ }^{3}$ \\ Zhiyong Yuan' \\ Ping Wang'
}

'Department of Radiotherapy, Tianjin Medical University Cancer Institute and Hospital, National Clinical Research Center for Cancer, Key Laboratory of Cancer Prevention and Therapy, Tianjin's Clinical Research Center for Cancer, Tianjin, ${ }^{2}$ Cyberknife Center, Ruikang Hospital, Guangxi Traditional Chinese Medical University, Nanning, ${ }^{3}$ Department of Radiotherapy, Shanghai Changhai Hospital, Second Military Medical University, Shanghai, People's Republic of China

*These authors contributed equally to this work
This article was published in the following Dove Press journal:

OncoTargets and Therapy

6 November 2017

Number of times this article has been viewed

Objective: The objective of this study was to investigate the safety of robotic stereotactic body radiation therapy (SBRT) for hepatocellular carcinoma (HCC) patients and its related factors. Methods: A total of 74 HCC patients with Child-Turcotte-Pugh (CTP) Class A were included in a multi-institutional, single-arm Phase II trial (NCT 02363218) between February 2013 and August 2016. All patients received SBRT treatment at a dose of $45 \mathrm{~Gy} / 3 \mathrm{f}$. The liver function was compared before and after SBRT treatment by the analysis of adverse hepatic reactions and changes in CTP classification.

Results: After SBRT treatment, eight patients presented with decreases in CTP classification and 13 patients presented with $\geq$ grade 2 hepatic adverse reactions. For patients presenting with $\geq$ grade 2 hepatic adverse reactions, the total liver volume of $\leq 1,162 \mathrm{~mL}$ and a normal liver volume (total liver volume - gross tumor volume [GTV]) of $\leq 1,148 \mathrm{~mL}$ were found to be independent risk factors and statistically significant $(P<0.05)$.

Conclusion: The total liver volume and normal liver volume are associated with the occurrence of $\geq$ grade 2 hepatic adverse reactions after SBRT treatment on HCC patients. Therefore, if the fractionated scheme of $45 \mathrm{~Gy} / 3 \mathrm{f}$ is applied in SBRT for HCC patients, a total liver volume $>1,162 \mathrm{~mL}$ and a normal liver volume $>1,148 \mathrm{~mL}$ should be ensured to improve therapeutic safety.

Keywords: hepatocellular carcinoma, robotic stereotactic body radiation therapy, CyberKnife, Child-Turcotte-Pugh class, adverse hepatic reactions

\section{Introduction}

Hepatocellular carcinoma (HCC) is one of the common malignant tumors. ${ }^{1}$ According to the practice guideline by American Association for the Study of Liver Diseases (AASLD), surgical resection, liver transplantation and treatment with percutaneous puncture are effective approaches for patients with early stage liver cancer. ${ }^{2}$ Recent advances in CyberKnife (Accuray Incorporated, Sunnyvale, CA, USA) stereotactic body radiation therapy (SBRT) technology not only enable highly focused dose delivery but also facilitate precise tumor localization with real-time image tracking and correction during treatment. With these techniques, SBRT can deliver a highly focused ablative dose to the tumor and accordingly achieve effective tumor control while minimizing the radiation-induced toxicity to normal tissue. ${ }^{3}$ However, radiation therapy for HCC patients is still limited mainly by radiation-induced liver disease (RILD) as the main complication. Currently, there are few reports on the analysis of factors affecting RILD after SBRT treatment for HCC patients. Thus, the safety and efficacy of SBRT on HCC patients were carried out by multicenter prospective study
Correspondence: Ping Wang

Department of Radiotherapy,Tianjin Medical University Cancer Institute and Hospital, National Clinical Research Center for Cancer, Key Laboratory of

Cancer Prevention and Therapy, Tianjin's Clinical Research Center for Cancer, Huan-Hu-Xi Road, Ti-Yuan-Bei, He Xi District, Tianjin, 300060, People's Republic of China

Email wangping@tjmuch.com 
and the factors relating to the hepatic adverse reactions after SBRT were investigated.

\section{Materials and methods}

The inclusion criteria were as follows. 1) Confirmed HCC according to one of the three European Association for the Study of the Liver criteria: i) histopathology; ii) two diagnostic imaging techniques (ultrasound [US], magnetic resonance imaging [MRI], computed tomography [CT], angiography) with the confirmation of a lesion $>2 \mathrm{~cm}$ accompanying the arterial hypervascularization and iii) one diagnostic imaging technique with confirmation of a lesion $>2 \mathrm{~cm}$ with arterial hypervascularization and an AFP $>400 \mathrm{ng} / \mathrm{mL}$. 2) Unifocal liver tumors not to exceed $5 \mathrm{~cm}$ in the greatest axial dimension. Multifocal lesions will be restricted to a maximum of three lesions with a maximum lesion size of $3 \mathrm{~cm}$ for each lesion, which can be treated within a single target volume within the same liver segment as long as the dose constraints to normal tissue can be met. 3) Volume of uninvolved liver $>750 \mathrm{~cm}^{3}$. 4) Hepatic lesion in patients for whom surgical resection is not possible or patients who refuse surgery. 5) Eastern Clinical Oncology Group (ECOG) performance status 0,1 or 2. 6) Patients with liver disease classified as Child-Pugh class A. 7) Life expectancy $>6$ months. 8) Age $>18$ years. 9) Albumin $>2.5 \mathrm{~g} / \mathrm{dL}$. 10) Total bilirubin $<3 \mathrm{mg} /$ dL. 11) international normalized ratio <1.5. 12) Transaminases (alanine transaminase and aspartate transaminase) no more than three times the upper limit of normal. 13) Creatinine $<2.0 \mathrm{mg} / \mathrm{dL}$. 14) Both men and women and members of all races and ethnic groups are eligible for this study. 15) Ability of the research subject or authorized legal representative to understand and the willingness to sign a written informed consent document. This multicenter study has been approved by the medical ethics committee of Tianjin Medical University Cancer Institute and Hospital, the E2013139A ethics guidelines of the committee were followed and written informed consents have been obtained from all enrolled patients. All institutions adopted the same inclusion criteria and the same treatment scheme and protocols. The principle investigator (PI) institution monitored all data enrolled. With the study protocol, SBRT was delivered to patients with the fractionated scheme of $45 \mathrm{~Gy} / 3 \mathrm{f}$. The follow-up was done at 1, 3, 6, 12, 18 and 24 months after SBRT with laboratory tests (involving blood cell counts, blood biological markers and tumor markers) and imaging examinations to assess the changes in Child-Turcotte-Pugh (CTP) classification in patients, and adverse reactions were assessed according to the National Cancer Institute Common Terminology Criteria for Adverse Events version 4.02 (NCI CTCAE v 4.02). ${ }^{4}$
SPSS 23.0 was used for the statistical analysis. All dosimetric measurements were expressed as the mean \pm standard deviation $(\overline{\mathrm{x}} \pm \mathrm{s})$ and median. The chi-square test and independent $t$-test were applied for the analysis on clinical characteristics and dosimetric parameters related to the changes in CTP classification and the occurrence of hepatic adverse reactions. Moreover, the logistic regression was used to analyze the independent factors affecting on liver functions. The receiver operating characteristic (ROC) curve was applied to assess the factors related to liver injury. $P<0.05$ was considered to be statistically significant.

\section{Results}

\section{Patients}

Between February 2013 and August 2016, a total of 74 HCC patients with CTP class A were included and completed SBRT treatment. The patients' characteristics are shown in Table 1.

Table I Characteristics of $74 \mathrm{HCC}$ patients

\begin{tabular}{|c|c|}
\hline Characteristics & Number of cases (\%) \\
\hline \multicolumn{2}{|l|}{ Sex } \\
\hline Male & $56(75.7)$ \\
\hline Female & $18(24.3)$ \\
\hline \multicolumn{2}{|l|}{ Age (years) } \\
\hline$\leq 60$ & $40(54.1)$ \\
\hline$>60$ & $34(45.9)$ \\
\hline \multicolumn{2}{|l|}{ ECOG scale } \\
\hline 0 and $I$ scores & $70(94.6)$ \\
\hline 2 score & $4(5.4)$ \\
\hline \multicolumn{2}{|c|}{ History of hepatitis B } \\
\hline Yes & $62(83.8)$ \\
\hline No & $12(16.2)$ \\
\hline \multicolumn{2}{|l|}{ BCLC staging } \\
\hline 0 & $15(20.3)$ \\
\hline A & $59(79.7)$ \\
\hline \multicolumn{2}{|l|}{ Prior TACE } \\
\hline Yes & $12(16.2)$ \\
\hline No & $62(83.8)$ \\
\hline \multicolumn{2}{|l|}{ GTV (mL) } \\
\hline$\leq 7.5$ & $14(18.9)$ \\
\hline$>7.5$ & $60(8 I .1)$ \\
\hline \multicolumn{2}{|c|}{ Total liver volume (mL) } \\
\hline$\leq \mathrm{I}, \mathrm{I} 62$ & $25(33.8)$ \\
\hline$>1,162$ & $49(66.2)$ \\
\hline \multicolumn{2}{|c|}{ Normal liver volume $(\mathrm{mL})$} \\
\hline$\leq \mathrm{I}, \mathrm{I} 48$ & $29(39.2)$ \\
\hline$>1,148$ & $45(60.8)$ \\
\hline \multicolumn{2}{|l|}{ Number of lesions } \\
\hline I & $67(90.5)$ \\
\hline$>1$ & $7(9.5)$ \\
\hline \multicolumn{2}{|c|}{ Intrahepatic progression } \\
\hline Yes & $12(16.2)$ \\
\hline No & $62(83.8)$ \\
\hline
\end{tabular}

Abbreviations: BCLC, Barcelona Clinic Liver Cancer; ECOG, Eastern Cooperative Oncology Group; GTV, gross tumor volume; HCC, hepatocellular carcinoma; TACE, transarterial chemoembolization. 
All enrolled patients had completed SBRT treatment and were in well compliance to the treatment. The 1-year and 2-year local control rates were $95.5 \%$ and $82.7 \%$, respectively. The 1-year and 2-year hepatic control (progressionfree interval) rates were $90.6 \%$ and $72.6 \%$, respectively. The 1-year and 2-year overall survival (OS) rates were 93.8\% and $91.5 \%$, respectively. The enrolled patients with signed informed consent forms underwent enhanced CT cans and MRI scans for radiation treatment planning and target delineation. CyberKnife SBRT with Synchrony Respiratory Tracking System was applied with at least one fiducial implantation. The fiducial was implanted as close to the lesion as possible with US guidance. The planning target volume was considered as the region $5 \mathrm{~mm}$ outside of clinical target volume, and the prescription isodose line was $>80 \%$ and covered at least $95 \%$ of planning target volume. The fractionation scheme was $45 \mathrm{~Gy}$ in three fractions. The treatment was completed within 1 week, and the follow-up period was 2 years. The median gross tumor volume (GTV) was $24.17 \mathrm{~mL}(2.13-123.6 \mathrm{~mL})$; the median liver volume was $1,295.9 \mathrm{~mL}(777.9-2,025.2 \mathrm{~mL})$; and the median normal liver volume was $1,274.00 \mathrm{~mL}(772.0$ $1,972.62 \mathrm{~mL}$ ). The detailed dosimetric parameters (involving the percentage of normal liver volume receiving 5, 15, 20, 25 and $30 \mathrm{~Gy}$ ) $\mathrm{V}_{5}, \mathrm{~V}_{15}, \mathrm{~V}_{20}, \mathrm{~V}_{25}$ and $\mathrm{V}_{30}$, respectively, in the total normal liver volume are shown in Table 2.

\section{Adverse reactions}

Within 3 months after SBRT, 13 patients presented with grades $2-3$ hepatic adverse reactions related to therapy. In all, 10 patients were with grade 2 side effects and three patients were with grade 3 side effects, of whom, one patient presented with syndromes of jaundice, asthenia and hepatic encephalopathy and had improved after hepatoprotective treatment; one presented with ascites, which was alleviated after hepatoprotective treatment; one presented with serious

Table 2 Dosimetric parameters in 74 HCC patients

\begin{tabular}{lll}
\hline Dosimetric parameters & Mean & Median \\
\hline GTV $(\mathrm{mL})$ & $31.08 \pm 27.69$ & 25.17 \\
Total liver volume $(\mathrm{mL})$ & $1,293.28 \pm 270.67$ & $1,268.40$ \\
Normal liver volume $(\mathrm{mL})$ & $1,243.72 \pm 265.22$ & $1,246.29$ \\
$\mathrm{~V}_{5}(\%)$ & $60.70 \pm 21.52$ & 61.34 \\
$\mathrm{~V}_{10}(\%)$ & $35.61 \pm 18.52$ & 32.19 \\
$\mathrm{~V}_{15}(\%)$ & $21.67 \pm 13.66$ & 17.79 \\
$\mathrm{~V}_{20}(\%)$ & $14.24 \pm 9.27$ & 11.32 \\
$\mathrm{~V}_{25}(\%)$ & $10.14 \pm 6.86$ & 8.07 \\
$\mathrm{~V}_{30}(\%)$ & $7.46 \pm 5.35$ & 6.09
\end{tabular}

Note: $\mathrm{V}_{5}, \mathrm{~V}_{10}, \mathrm{~V}_{15}, \mathrm{~V}_{20}, \mathrm{~V}_{25}$, and $\mathrm{V}_{30}$ indicate detailed dosimetric parameters involving the percentage of normal liver volume receiving 5, 10, 15, 20, 25, and $30 \mathrm{~Gy}$ respectively.

Abbreviations: GTV, gross tumor volume; HCC, hepatocellular carcinoma ascites at 4 months after therapy and then died due to spontaneous peritonitis; 10 cases manifested transaminase elevation, nausea, vomiting, lost appetite and abdominal pain, which were relieved after symptomatic treatment. No patients presented with $>$ grade 4 adverse reactions. The CTP assessment showed that eight patients presented with the decreased CTP on its classification after radiation therapy, of whom, one patient experienced a decrease from CTP Class A to CTP Class $\mathrm{C}$ and the other seven patients presented with decreases from CTP Class A to CTP Class B.

The independent $t$-test and chi-square test were used to analyze the dosimetric parameters and patients' characteristics related to the changes in CTP classification and the hepatic adverse reactions after radiation therapy among the 74 HCC patients. The results showed that both total liver volume and normal liver volume were statistically significant for the occurrence of $\geq$ grade 2 hepatic adverse reactions after SBRT treatment $(P<0.05$; Tables 3 and 4$)$.

The aforementioned results with the logistic regression analysis showed that both total liver volume and normal liver volume were independent risk factors for the occurrence of hepatic adverse reactions after SBRT treatment $(P<0.05$; Table 5).

ROC curves for the total liver volume and normal liver volume were used to predict the tolerance volume for SBRT on the enrolled 74 HCC patients (Figures 1 and 2). Figure 1 shows that the area under the curve was 0.718 (95\% confidence interval [CI]: $0.569-0.866$ ), the optimal cutoff point according to the Youden's index was $1,162 \mathrm{~mL}$, the sensitivity was $73.0 \%$ and the specificity was $69.2 \%$. Among the 74 HCC patients, the incidence of grades 2-3 adverse reactions in patients with a total liver volume $\leq 1,162 \mathrm{~mL}$ was $36.0 \%(9 / 25)$ vs $8.16 \%(4 / 49)$ in those with a total liver volume $>1,162 \mathrm{~mL}$, and the difference was statistically significant $(P<0.05)$. Figure 2 shows that the area under the curve was 0.700 (95\% CI: 0.543-0.856) and the optimal cutoff point was $1,148 \mathrm{~mL}$, the sensitivity was $66.7 \%$ and the specificity was $69.2 \%$. Among the 74 HCC patients, the incidence of grades 2-3 adverse reactions in patients with a normal liver volume $\leq 1,148 \mathrm{~mL}$ was $31.0 \%(9 / 29)$ vs $8.89 \%(4 / 45)$ in those with a normal liver volume $>1,148 \mathrm{~mL}$, and the difference was statistically significant $(P<0.05)$.

\section{Discussion}

With the advances in computer imaging techniques and SBRT as well as their incessant applications in the treatment for liver cancer, ${ }^{5-10}$ SBRT has been widely considered as a safe and an effective technique for the treatment of 
Table 3 Analysis of dosimetric parameters, changes in CTP classification and the occurrence of hepatic adverse reactions in $74 \mathrm{HCC}$ patients

\begin{tabular}{|c|c|c|c|c|c|c|}
\hline \multirow{2}{*}{$\begin{array}{l}\text { Dosimetric } \\
\text { parameters }\end{array}$} & \multicolumn{2}{|c|}{ Changes in CTP classification } & \multirow[t]{2}{*}{$P$-value } & \multicolumn{2}{|c|}{ Hepatic adverse reactions } & \multirow[t]{2}{*}{$P$-value } \\
\hline & No & Yes & & Grades 0-I & Grades 2-3 & \\
\hline GTV (mL) & $32.83 \pm 27.98$ & $25.93 \pm 26.13$ & 0.510 & $32.82 \pm 28.84$ & $28.62 \pm 22.19$ & 0.623 \\
\hline Total liver volume (mL) & $1,297.11 \pm 263.19$ & $1,216.67 \pm 345.72$ & 0.729 & $\mathrm{I}, 327.79 \pm 273.34$ & $I,|3| .31 \pm 194.03$ & 0.016 \\
\hline Normal liver volume $(\mathrm{mL})$ & $1,246.97 \pm 255.86$ & $1,217.35 \pm 353.27$ & 0.768 & $\mathrm{I}, 277.22 \pm 265.37$ & $\mathrm{I}, 086.82 \pm 208.44$ & 0.018 \\
\hline $\mathrm{V}_{5}(\%)$ & $61.23 \pm 21.30$ & $56.36 \pm 24.31$ & 0.549 & $60.01 \pm 21.50$ & $63.96 \pm 22.15$ & 0.552 \\
\hline $\mathrm{V}_{10}(\%)$ & $35.04 \pm 18.65$ & $32.12 \pm 18.20$ & 0.576 & $35.15 \pm 18.64$ & $37.80 \pm|8.5|$ & 0.642 \\
\hline$V_{15}(\%)$ & $22.00 \pm 13.88$ & $18.87 \pm 12.02$ & 0.543 & $21.35 \pm 13.69$ & $23.18 \pm 13.94$ & 0.664 \\
\hline $\mathrm{V}_{20}(\%)$ & $|4.4| \pm 9.39$ & $12.88 \pm 8.60$ & 0.662 & $13.86 \pm 9.03$ & $16.04 \pm 10.5 \mid$ & 0.445 \\
\hline $\mathrm{V}_{25}(\%)$ & $10.25 \pm 6.95$ & $9.23 \pm 6.37$ & 0.695 & $9.90 \pm 6.85$ & 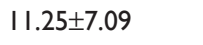 & 0.524 \\
\hline $\mathrm{V}_{30}(\%)$ & $7.56 \pm 5.43$ & $6.62 \pm 4.83$ & 0.642 & $7.39 \pm 5.49$ & $7.8 I \pm 4.86$ & 0.800 \\
\hline
\end{tabular}

Note: $\mathrm{V}_{5}, \mathrm{~V}_{10}, \mathrm{~V}_{15}, \mathrm{~V}_{20}, \mathrm{~V}_{25}$, and $\mathrm{V}_{30}$ indicate detailed dosimetric parameters involving the percentage of normal liver volume receiving 5, I0, I5, 20, 25, and 30 Gy, respectively. Abbreviations: CTP, Child-Turcotte-Pugh; GTV, gross tumor volume; HCC, hepatocellular carcinoma.

Table 4 Analysis of patients' characteristics in CTP classification and the occurrence of hepatic adverse reactions in 74 HCC patients

\begin{tabular}{|c|c|c|c|c|c|c|c|c|}
\hline \multirow[t]{2}{*}{ Characteristics } & \multirow[t]{2}{*}{$\begin{array}{l}\text { Patients } \\
\text { (n) }\end{array}$} & \multicolumn{2}{|c|}{$\begin{array}{l}\text { Changes in CTP } \\
\text { classification }\end{array}$} & \multirow[t]{2}{*}{$P$-value } & \multirow[t]{2}{*}{$\begin{array}{l}\text { Patients } \\
\text { (n) }\end{array}$} & \multicolumn{2}{|c|}{ Hepatic adverse reactions } & \multirow[t]{2}{*}{$P$-value } \\
\hline & & No & $\overline{\text { Yes }}$ & & & Grades 0-I & Grades 2-3 & \\
\hline \multicolumn{4}{|l|}{$\overline{\text { Sex }}$} & 0.697 & & & & 0.810 \\
\hline Male & 56 & 49 & 7 & & 56 & 47 & 9 & \\
\hline Female & 18 & 17 & I & & 18 & 14 & 4 & \\
\hline \multicolumn{4}{|l|}{ Age (years) } & 1.000 & & & & 0.987 \\
\hline$\leq 60$ & 40 & 36 & 4 & & 40 & 33 & 7 & \\
\hline$>60$ & 34 & 30 & 4 & & 34 & 28 & 6 & \\
\hline \multicolumn{4}{|l|}{ ECOG scale } & 0.911 & & & & 1.000 \\
\hline 0 and I scores & 70 & 63 & 7 & & 70 & 58 & 12 & \\
\hline 2 score & 4 & 3 & I & & 4 & 3 & I & \\
\hline \multicolumn{4}{|c|}{ History of hepatitis B } & 1.000 & & & & 0.745 \\
\hline Yes & 62 & 55 & 7 & & 62 & 52 & 10 & \\
\hline No & 12 & II & I & & 12 & 9 & 3 & \\
\hline \multicolumn{4}{|l|}{ BCLC staging } & 0.080 & & & & 1.000 \\
\hline 0 & 15 & 11 & 4 & & 15 & 12 & 3 & \\
\hline$A$ & 59 & 55 & 4 & & 59 & 49 & 10 & \\
\hline \multicolumn{4}{|l|}{ Prior TACE } & 0.837 & & & & 0.929 \\
\hline Yes & 12 & 10 & 2 & & 12 & 10 & 2 & \\
\hline No & 62 & 56 & 6 & & 62 & 51 & 11 & \\
\hline \multicolumn{4}{|l|}{ GTV (mL) } & 0.058 & & & & 1.000 \\
\hline$\leq 7.5$ & 14 & 10 & 4 & & 14 & 12 & 2 & \\
\hline$>7.5$ & 60 & 56 & 4 & & 60 & 49 & 11 & \\
\hline \multicolumn{4}{|c|}{ Total liver volume $(\mathrm{mL})$} & 0.155 & & & & 0.008 \\
\hline$\leq 1,162$ & 25 & 20 & 5 & & 25 & 16 & 9 & \\
\hline$>1,162$ & 49 & 46 & 3 & & 49 & 45 & 4 & \\
\hline \multicolumn{4}{|c|}{ Normal liver volume $(\mathrm{mL})$} & 0.295 & & & & 0.015 \\
\hline$\leq 1,148$ & 29 & 24 & 5 & & 29 & 20 & 9 & \\
\hline$>1,148$ & 45 & 42 & 3 & & 45 & 41 & 4 & \\
\hline \multicolumn{4}{|l|}{ Number of lesions } & 1.000 & & & & 0.185 \\
\hline 1 & 67 & 60 & 7 & & 67 & 57 & 10 & \\
\hline$>1$ & 7 & 6 & I & & 7 & 4 & 3 & \\
\hline \multicolumn{4}{|c|}{ Intrahepatic progression } & 0.837 & & & & 0.614 \\
\hline Yes & 12 & 10 & 2 & & 12 & II & I & \\
\hline No & 62 & 56 & 6 & & 62 & 50 & 12 & \\
\hline
\end{tabular}

Abbreviations: BCLC, Barcelona Clinic Liver Cancer; CTP, Child-Turcotte-Pugh; ECOG, Eastern Cooperative Oncology Group; GTV, gross tumor volume; HCC, hepatocellular carcinoma; TACE, transarterial chemoembolization. 
Table 5 Logistic regression analysis of related factors affecting $\geq$ grade 2 hepatic adverse reactions after SBRT treatment

\begin{tabular}{lllllll}
\hline Variables & $\boldsymbol{\beta}$ & SE & Wald & $\boldsymbol{P}$-value & $\mathbf{R R}$ & $\mathbf{9 5 \%} \mathbf{C l}$ \\
\hline Sex & 0.400 & 0.674 & 0.353 & 0.553 & 1.492 & $0.398-5.587$ \\
Age (years) & 0.010 & 0.613 & 0.085 & 0.987 & 1.010 & $0.304-3.353$ \\
ECOG scale & 0.477 & 1.197 & 0.159 & 0.690 & 1.611 & $0.154-16.842$ \\
History of hepatitis B & 0.550 & 0.751 & 0.537 & 0.464 & 0.577 & $0.132-2.513$ \\
BCLC staging & 0.203 & 0.733 & 0.077 & 0.782 & 0.816 & $0.194-3.433$ \\
Prior of TACE & 0.076 & 0.843 & 0.008 & 0.929 & 1.078 & $0.207-5.267$ \\
GTV (mL) & 0.298 & 0.833 & 0.128 & 0.721 & 1.347 & $0.263-6.899$ \\
Total liver volume $(\mathrm{mL})$ & -1.806 & 0.665 & 7.388 & 0.007 & 0.164 & $0.045-0.604$ \\
Normal liver volume $(\mathrm{mL})$ & -1.504 & 0.658 & 5.230 & 0.022 & 0.222 & $0.061-0.806$ \\
Number of lesions & 1.453 & 0.837 & 3.011 & 0.083 & 4.257 & $0.829-22.057$ \\
Intrahepatic progression & 0.083 & 0.722 & 0.013 & 0.908 & 0.920 & $0.223-3.790$ \\
\hline A & & &
\end{tabular}

Abbreviations: BCLC, Barcelona Clinic Liver Cancer; Cl, confidence interval; ECOG, Eastern Cooperative Oncology Group; GTV, gross tumor volume; RR, relative risk; SBRT, stereotactic body radiation therapy; SE, standard error; TACE, transarterial chemoembolization.

liver cancer. ${ }^{8,11-13}$ Our study focused on the factors affecting hepatic adverse reactions caused by radiation therapy and analyzed the clinical characteristics and dosimetric parameters for more suitable patients for SBRT treatment, thereby reducing the occurrence of adverse reactions induced by radiation therapy. ${ }^{14-16}$

The previous studies have illustrated that an SBRT dose of 24-60 Gy administrated in three to six fractions is safe and efficacious for the treatment of HCC. In the meanwhile, the normal liver tissue threshold dose and the volume irradiated are well correlated. ${ }^{17-20}$ The incidence of RILD becomes significantly higher when

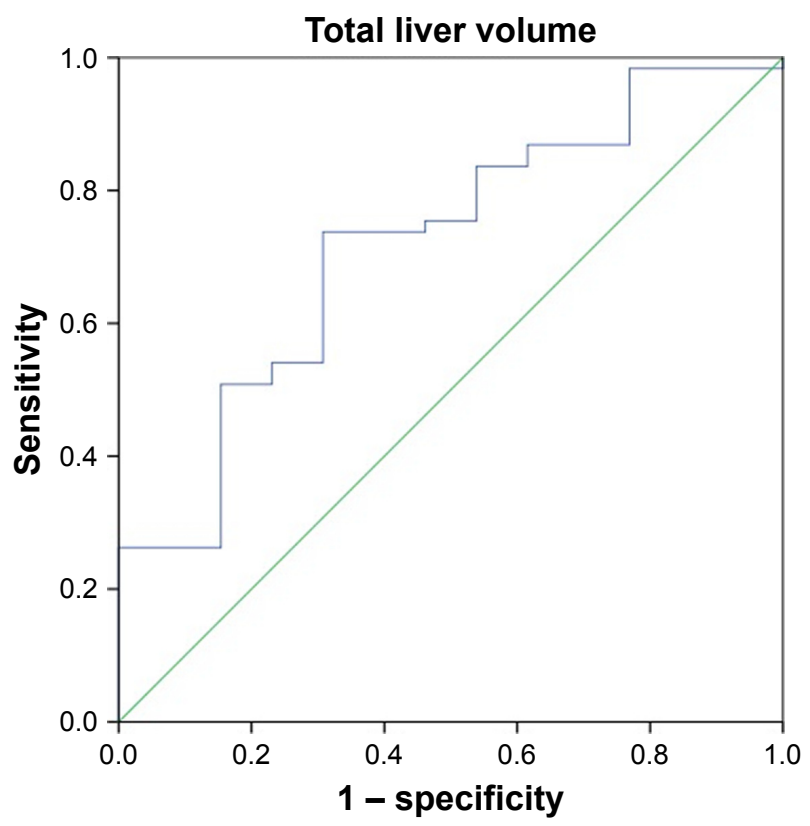

Figure I ROC curve for the total liver volume $(\mathrm{mL})$ predicting the occurrence of $\geq$ grade 2 hepatic adverse reactions after SBRT treatment.

Abbreviations: ROC, receiver operating characteristic; SBRT, stereotactic body radiation therapy. the mean hepatic dose reaches 23 Gy. ${ }^{17}$ Liang et al have analyzed 109 primary liver carcinomas treated with hypofractionated three-dimensional conformal radiation therapy (3D-CRT) and have found that for CTP class A patients, the hepatic radiation tolerance was with a mean dose to normal liver of 23 Gy. ${ }^{17}$ Since the coherent nature of SBRT techniques is with steeper dose gradients and more conformal isodose curve compared to the conventional radiotherapy technology and 3D-CRT technology, a higher, safer and more accurate dose exposure could be delivered to the liver lesion area while keeping normal liver tissue and lesions' adjacent areas at a lower dose with SBRT techniques. Thus,

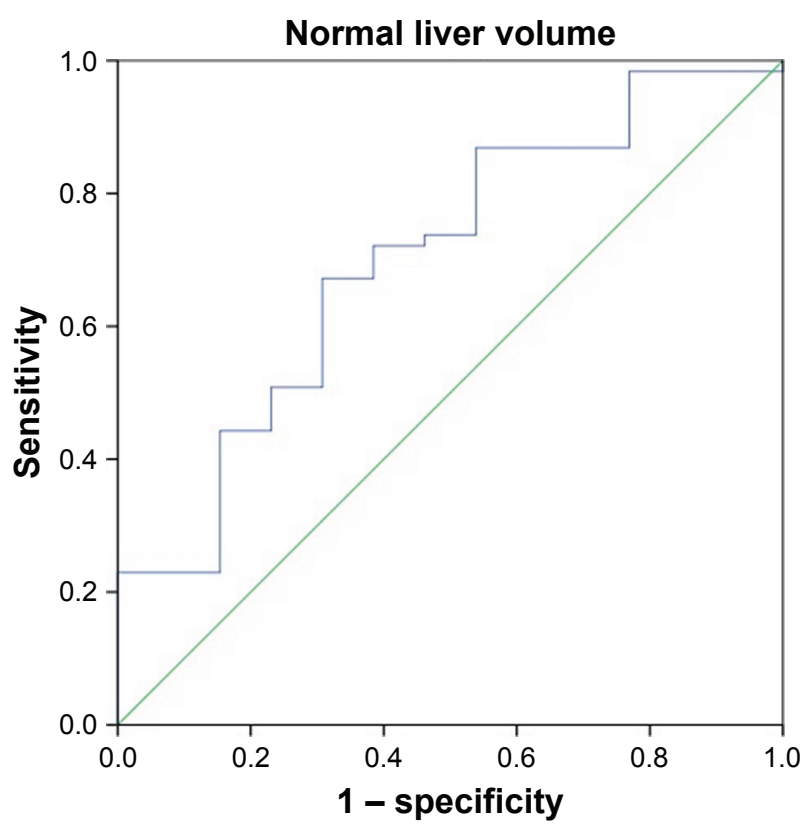

Figure 2 ROC curve for the normal liver volume $(\mathrm{mL})$ predicting the occurrence of $\geq$ grade 2 hepatic adverse reactions after SBRT treatment.

Abbreviations: ROC, receiver operating characteristic; SBRT, stereotactic body radiation therapy. 
we might further explore the relationship between the normal liver tissue threshold dose and the volume irradiated based on Liang et al's findings.

Our dose scheme of $45 \mathrm{~Gy} / 3 \mathrm{f}$ to HCC with CTP class A was in line with the previous study done by Kwon et $\mathrm{al}^{21}$ on the treatment scheme and has achieved the same therapeutic effect with no grade 4 liver toxicity. In addition, the inclusion criteria of patients with one to three hepatic lesions and maximum individual tumor diameters $<5 \mathrm{~cm}$ in our study were in line with the previous work done by Rusthoven et al, ${ }^{8}$ in which unifocal liver tumors were not to exceed $5 \mathrm{~cm}$ in the greatest axial dimension and multifocal lesions were restricted to a maximum of three lesions with a maximum lesion size of $3 \mathrm{~cm}$ for each lesion. In such tumor volume, the treatment scheme of $45 \mathrm{~Gy} / 3 \mathrm{f}$ was proved to be safe and efficient.

With the same treatment scheme, Andolino et $\mathrm{al}^{22}$ also evaluated the safety and efficacy of SBRT for the treatment of $\mathrm{HCC}$ and found no $\geq$ grade 3 non-hematologic toxicities, although $13 \%$ of patients experienced an increase in hematologic and hepatic injuries greater than 1 grade and $20 \%$ experienced progression in CTP class within 3 months of treatment.

Son et $\mathrm{al}^{16}$ reported that total liver volume receiving 18 Gy should be $800 \mathrm{~cm}^{3}$ to reduce the risk of deterioration of hepatic function and suggests that liver volume may be related to the occurrence of radiation-induced hepatic toxicity. In their study, the total dose administered was 30-39 Gy (median, 36 Gy) by three to five fractions while keeping $\leq 15 \mathrm{~Gy} / \mathrm{f}$. The progression of CTP class after SBRT limits other additional local treatments and also reflects the deterioration of hepatic function. Therefore, it would be important to note that the presence or absence of the progression of CTP class is a dose-limiting factor. The total liver volume receiving $<18$ Gy should be $>800 \mathrm{~cm}^{3}$ to reduce the risk of the deterioration of hepatic function.

A study by Pan et al found that the occurrence of hepatic adverse reactions after SBRT for liver cancer was associated with dose-volume parameters. For patients with HCC, it is recommended that the normal liver volume is $\geq 700 \mathrm{cc}$ with the single-fraction dose of $\leq 15 \mathrm{~Gy}$. In the meantime, the constrains of $\mathrm{D}_{33}<21$ Gy and $\mathrm{D}_{50}<15$ Gy were taken as the standard when three to five fractionated SBRT treatment schemes were applied. ${ }^{6}$ In our study, the uniform inclusion criteria and prescribed doses enable a statistical analysis of clinical characteristics and dosimetric parameters; the results showed that both total liver volume and normal liver volume were independent risk factors affecting the occurrence of $\geq$ grade 2 hepatic adverse reactions after radiation therapy. Based on our fractionated dose scheme of $45 \mathrm{~Gy} / 3 \mathrm{f}$ for $\mathrm{HCC}$ patients, a total liver volume $>1,162 \mathrm{~mL}$ and a normal liver volume $>1,148 \mathrm{~mL}$ should be ensured to improve the therapeutic safety. GTV is an independent predictor for prior treatment on CTP worsening $(P=0.058)$. It seems that the smaller the GTV, the better the prognosis could be achieved. ${ }^{23}$

The progress of CTP and RIRD occurrence in HCC patients after SBRT is an important predictor factor of treatment safety; thus, patients with CTP for A were enrolled in our study and factors affecting the CTP progress and the $\geq$ grade 2 hepatic adverse reactions occurrence after SBRT treatment were analyzed. We explored on the correlation between adverse effect of $\geq$ grade 2 and the total liver volume as well as the normal liver volume and drew the conclusion that if the fractionated scheme of $45 \mathrm{~Gy} / 3 \mathrm{f}$ is applied in SBRT for HCC patients, a total liver volume $>1,162 \mathrm{~mL}$ and a normal liver volume $>1,148 \mathrm{~mL}$ should be ensured to improve therapeutic safety.

There are some limitations in our study. First, the single-arm study has the inclusion criteria on CTP classification, tumor sizes and the normal liver volume as well as total liver volume; thus, it is not indicated for all HCC patients. Second, some enrolled patients (27/74) had received prior treatments such as transarterial chemoembolization, radiofrequency ablation and surgery, which may affect the patients' compliance to SBRT and bias the current results. The open-label study with a large sample size is needed to further investigate.

\section{Conclusion}

We established a model for the relationship between the occurrence of hepatic adverse reactions to SBRT for HCC patients and the liver volume. We considered that both total liver volume and normal liver volume were independent risk factors affecting the occurrence of $\geq$ grade 2 hepatic adverse reactions after SBRT treatment. For fractionated scheme of $45 \mathrm{~Gy} / 3 \mathrm{f}$, a total liver volume of $>1,162 \mathrm{~mL}$ and a normal liver volume of $>1,148 \mathrm{~mL}$ should be ensured to improve the therapeutic safety.

\section{Acknowledgment}

The authors acknowledge Dr Jiuhong Chen for her valuable advice on revision of the manuscript.

\section{Disclosure}

The authors report no conflicts of interest in this work. 


\section{References}

1. Torre LA, Bray F, Siegel RL, Ferlay J, Lortet-Tieulent J, Jemal A. Global cancer statistics, 2012. CA Cancer J Clin. 2015;65(2):87-108.

2. Bruix J, Sherman M. Management of hepatocellular carcinoma. Hepatology. 2005;42(5):1208-1236.

3. Feng M, Ben-Josef E. Radiation therapy for hepatocellular carcinoma. Semin Radiat Oncol. 2011;21(4):271-277.

4. Clinical Trials. Tianjin Medical University Cancer Institute and Hospital: Study on effectiveness and safety of hepatocellular carcinoma patients treated with CyberKnife: Trial record 1 of 1 for: NCT02363218 [update October 5, 2016; cited May 18, 2017]. Available from: https://clinicaltrials. gov/ct2/show/NCT02363218?term=NCT02363218\&rank=1. NLM identifier: NCT02363218. Accessed May 20, 2017.

5. Bujold A, Massey CA, Kim JJ, et al. Sequential phase I and II trials of stereotactic body radiotherapy for locally advanced hepatocellular carcinoma. J Clin Oncol. 2013;31(13):1631-1639.

6. Pan CC, Kavanagh BD, Dawson LA, et al. Radiation-associated liver injury. Int J Radiat Oncol Biol Phys. 2010;76(3 suppl):S94-S100.

7. Cao Y, Wang H, Johnson TD, et al. Prediction of liver function by using magnetic resonance-based portal venous perfusion imaging. Int J Radiat Oncol Biol Phys. 2013;85(1):258-263.

8. Rusthoven KE, Kavanagh BD, Cardenes H, et al. Multi-institutional phase I/II trial of stereotactic body radiation therapy for liver metastases. J Clin Oncol. 2009;27(10):1572-1578.

9. Høyer M, Swaminath A, Bydder S, et al. Radiotherapy for liver metastases: a review of evidence. Int J Radiat Oncol Biol Phys. 2012;82(3): 1047-1057.

10. Goodman KA, Wiegner EA, Maturen KE, et al. Dose-escalation study of single-fraction stereotactic body radiotherapy for liver malignancies. Int J Radiat Oncol Biol Phys. 2010;78(2):486-493.

11. Eriguchi T, Takeda A, Sanuki N, et al. Acceptable toxicity after stereotactic body radiation therapy for liver tumors adjacent to the central biliary system. Int J Radiat Oncol Biol Phys. 2013;85(4):1006-1011.

12. van der Pool AEM, Méndez Romero A, Wunderink W, et al. Stereotactic body radiation therapy for colorectal liver metastases. Br J Surg. 2010;97(3):377-382.
13. Wulf J, Guckenberger M, Haedinger U, et al. Stereotactic radiotherapy of primary liver cancer and hepatic metastases. Acta Oncol. 2006;45(7): 838-847.

14. Dawson LA, Haken Ten RK, Lawrence TS. Partial irradiation of the liver. Semin Radiat Oncol. 2001;1:240-246.

15. Guha C, Kavanagh BD. Hepatic radiation toxicity: avoidance and amelioration. Semin Radiat Oncol. 2011;21(4):256-263.

16. Son SH, Choi BO, Ryu MR, et al. Stereotactic body radiotherapy for patients with unresectable primary hepatocellular carcinoma: dosevolumetric parameters predicting the hepatic complication. Int J Radiat Oncol Biol Phys. 2010;78(4):1073-1080.

17. Liang SX, Zhu XD, Xu ZY, et al. Radiation-induced liver disease in three-dimensional conformal radiation therapy for primary liver carcinoma: the risk factors and hepatic radiation tolerance. Int J Radiat Oncol Biol Phys. 2006;65(2):426-434.

18. Cheng JC, Wu JK, Huang CM, et al. Radiation-induced liver disease after three-dimensional conformal radiotherapy for patients with hepatocellular carcinoma: dosimetric analysis and implication. Int J Radiat Oncol Biol Phys. 2002;54(1):156-162.

19. Lawrence TS, Robertson JM, Anscher MS, Jirtle RL, Ensminger WD, Fajardo LF. Hepatic toxicity resulting from cancer treatment. Int $J$ Radiat Oncol Biol Phys. 1995;31(5):1237-1248.

20. Park HC, Seong J, Han KH, Chon CY, Moon YM, Suh CO. Dose response relationship in local radiotherapy for hepatocellular carcinoma. Int J Radiat Oncol Biol Phys. 2002;54(1):150-155.

21. Kwon JH, Bae SH, Kim JY, et al. Long-term effect of stereotactic body radiation therapy for primary hepatocellular carcinoma ineligible for local ablation therapy or surgical resection. Stereotactic radiotherapy for liver cancer. BMC Cancer. 2010;10:1-10.

22. Andolino DL, Johnson CS, Maluccio M, et al. Stereotactic body radiotherapy for primary hepatocellular carcinoma. Int J Radiat Oncol Biol Phys. 2011;81(4):e447-e453.

23. Wada H, Takai Y, Nemoto K, Yamada S. Univariate analysis of factors correlated with tumor control probability of three-dimensional conformal hypofractionated high-dose radiotherapy for small pulmonary or hepatic tumors. Int J Radiat Oncol Biol Phys. 2004;58(4): 1114-1120.
OncoTargets and Therapy

\section{Publish your work in this journal}

OncoTargets and Therapy is an international, peer-reviewed, open access journal focusing on the pathological basis of all cancers, potential targets for therapy and treatment protocols employed to improve the management of cancer patients. The journal also focuses on the impact of management programs and new therapeutic agents and protocols on

\section{Dovepress}

patient perspectives such as quality of life, adherence and satisfaction. The manuscript management system is completely online and includes a very quick and fair peer-review system, which is all easy to use. Visit http://www.dovepress.com/testimonials.php to read real quotes from published authors. 